\title{
Temperature estimating method for exhaust gases in valveless pulsejet engine
}

The article describes the problem of measuring the temperature in a pulse combustion chamber. The object of the study is a valveless pulsejet. The problem is analysed on the example of exhaust gases temperature measurement. The measurement in these conditions requires the use of a sensor resistant to large changes in gas velocity and temperature and at the same time with adequatly low inertia. This excludes the use of fast and precise yet thin, resistant wire sensors or ultrafast thin film thermocouples. Finally, a temperature measurement system based on sheated thermocouples was chosen. During each test the thermocouple has its own temperature which is different from the medium temperature. In order to properly determine the measured temperature of flowing media it is necessary to take the sensor time characteristics into account. In this article the iteration method is proposed to solve this problem.

Key words: pulsating combustion, pulsejet, temperature measurement, periodic flow, combustion chamber

\section{Introduction}

Combustion driven oscillations were first observed in 1777. When small flame was moving inside a vertical tube in a certain location the sound was generated (so called singing tube) [10].

In the vast majority of combustion chambers currently used in the gas turbines in industry or aviation, in which combustion is carried out under constant pressure, oscillations caused by combustion are unfavorable. Numerous experiments have been carried out in order to find the way to prevent this, because it can lead to significant damage to the combustion chamber or the entire device in which the chamber operates.

There is also a group of pulsating combustion chambers in which combustion occurs periodically in isochoric manner. In those devices pressure pulsations are desirable. High pressure fluctuations in this case intensify combustion process and in consequence combustion efficiency is higher than in isobaric devices. The oldest representant of this group is a pulsejet engine.

First pulsejet engine was patented in 1907 by Russian engineer Karavodin [10]. Later, it was widely used by Germans during the Second World War to propel V-1 "buzz bomb". After the war various tests were conducted on pulsejet engines. Unfortunately, the engines' disadvantages such as high noise, vibration, low propulsive efficiency and narrow throttle ability made pulsejet engines useless for manned aircraft. In consequence pulse combustion research fell into decline.

Despite the complex nature of the pulsating combustion process, which is a result of not fully explained chemical and gasdynamic phenomena interaction, it has remained in the sphere of researcher's interest. In fact, it has been noted for some time now that the interest in pulsed combustion chambers has increased. The advantages of pulsating combustion are widely appreciated as the pulsating combustion chambers are implemented in impingement drying, gas boilers or used for propelling the Unmanned Aerial Vehicles (UAV). According to [4] they can be even used to produce fuels from sewage sludge.

Although their construction is simple, pulsating combustion chambers are considered to be extremely demanding as research objects. Tests conducted by authors of this article proved that even a minor change of geometrical configura- tion can lead to significant changes in engine behaviour. Moreover, the change in combustion chamber dimension during its work due to thermal expansion as well as high vibration have to be taken into account during the tests.

One of the most important measurements in the pulsed combustion research program is the temperature measurement in characteristic sections. Determining the temperature and fuel flow values allows one to estimate combustion efficiency, especially when an exhaust gas analyser is additionally used. To measure the temperature in pulsations conditions of the combustion process with a frequency exceeding $100 \mathrm{~Hz}$ in the tested engine and temperature values exceeding $1000 \mathrm{~K}$, it is necessary to create an appropriate and credible measuring system. An additional difficulty is caused by the explosive, unstable combustion process, during which a medium flow with high speed and temperature variations is generated. This requires the use of a sensor that, apart from the adequate mechanical strength, should be characterized by an appropriate response to rapid changes in the measured parameter.

Despite the difficulties with the selection of sensors that meet the required criteria, the temperature tests of exhaust gases temperature (EGT) in the outlet cross-section of the valveless pulsejet engine were carried out. Average as well as instantaneous values were tested.

Research was conducted on specially designed test bench placed in Institute of Heat Engineering of Faculty of Power and Aeronautical Engineering of Warsaw University of Technology. During the work, a measuring station containing all the modules needed for effective temperature measurement was designed and built. The sensor was verified in terms of its ability to record temperature changes during the engine's operating cycle and then the adequate measurements were made. Test results were analyzed using an application built in the LabView environment as well as by using Matlab software and Excel applications.

\section{Description of test stand}

The view of the test stand is presented in Fig. 1. The main element of the stand is a valveless pulsejet engine, which was placed on a frame made of aluminum profiles, thanks to which any configuration of its setting was possible, allowing optimal spacing of the sensors. 


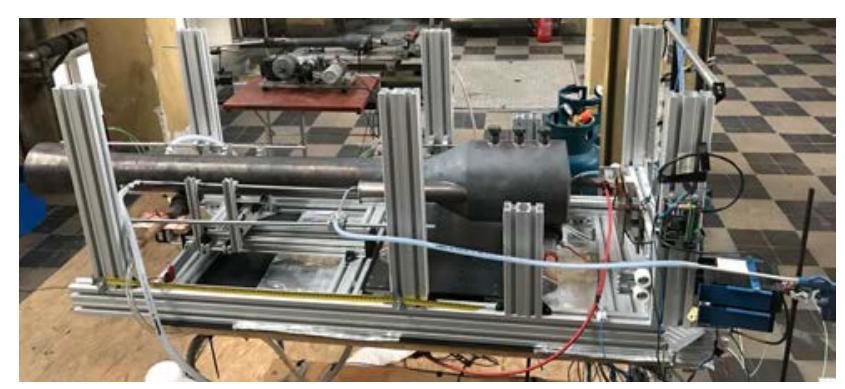

Fig. 1. The view of the test stand

The engine was attached to the stand at one point only. This was the center of the front wall of the combustion chamber and the point from which the thrust was simultaneously received. The other two supporting elements allowed the engine to move along them during its expansion as a result of the high temperature.

The engine test bench, apart from the standard elements allowing startup and running of the engine, was equipped with ultrafast Kistler pressure transducer assigned for combustion chamber actual pressure measurement. To avoid thermal damage to the sensor, it was equipped with a water cooling system and a temperature control system.

The tested pulsejet engine was fueled by propane gas. Fuel flow measurement was provided by rotameter with $4 \%$ accuracy. To increase the accuracy of rotameter readings depending on fuel pressure, two fuel pressure gauges were installed in the system - one at the inlet and one at the outlet of the meter. Additionally, to verify fuel flow readings a precise scale was used to weight the gas container before and after the engine run. To control gas fuel flow as precisely as possible propane bottles were equipped with a heating and temperature monitoring system. The thrust measurement was carried out by means of a tensometric beam connected to the measuring card using the necessary transducer.

To avoid thrust readings distortions a special force transformation system was designed to allow movement of sensor together with the engine horizontal axis when it was increasing its volume due to high temperature.

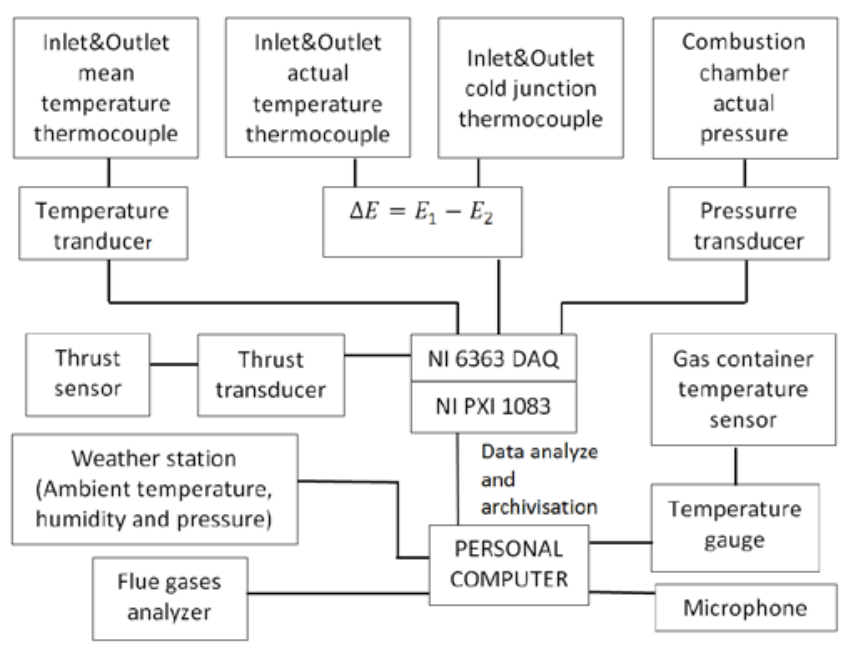

Fig. 2. Measurement system block diagram
To measure mean temperature in the outlet and inlet stations K-type thermocouples were used. A dynamic temperature measurement layout will be described further in this article.

Data acquisition was carried out using a PXI - 1082 computer with a 6363 data acquisition card. The acquisition, archiving and initial analysis of test data took place in the LabView environment. The block diagram of the entire measuring system is shown in Fig. 2.

\section{EGT in outlet station measurement}

\subsection{Selection of measuring method}

Temperature measurement in exhaust station of pulsejet engine is one of the pivotal elements of the pulse combustion research program. It not only provides information about combustion quality but it also becomes a significant value when verifying numerical simulations.

Despite the importance of this parameter, authors of this publication found it impossible to come across any work which would refer to the analyzed problem in the field of pulsating combustion research in a satisfactory manner. In works $[8,9]$, some trials are undertaken but they are only rough measurements of mean EGT without any detailed comments. Authors of [8] also emphasize difficulties during the tests of pulsejet engine.

Some advanced works $[5,6]$ were conducted by internal combustion engines researchers. In [5] authors pointed out that measurement of crank-angle resolved EGT is a challenging task because modern optical non-intrusive measurement methods requires substantial engine modifications and are consequently in few engine research laboratories. Authors provide precise EGT measurements but also concluded that this is only estimation and further work is needed. Similar work and conclusions were developed in [6]. But in this case there is no crank-angle resolved EGT estimation. It was concluded that further work is necessary to determine the method which ought to allow examination of high frequency cyclic fluctuations.

Other group of researchers was focused on thin film ultrafast thermocouples [1-3]. This kind of sensors seems to be the most promising because the time constant is of the order of ns. Moreover, the sensors are able to withstand temperatures up to $790^{\circ} \mathrm{C}$ [1]. Unfortunately, their application in harsh environment requires placing them on some surface [3]. This in turn causes increase in the time of reaction because the sensor is measuring the surface temperature, so in consequence the real time constant is dependent of thermal inertia of surface's material.

After taking into account different hardware solutions, a dynamic temperature measurement system based on sheathed thermocouples was chosen. Thermocouples are widely used in all kinds of combustion engines because their small sizes and reasonable time-constant allow for measurement during engine run. Additionally, in the application considered, the sensor could be destroyed easily. With thermocouples, it was possible to keep the cost of frequent sensor replacement at acceptable levels.

The K-type thermocouple used met the criteria for measuring the temperature in the engine outlet station. Its measuring range is from $-100^{\circ} \mathrm{C}$ to $1000^{\circ} \mathrm{C}$ for long-term 
measurements, while with temporary use the measuring range is from $-200^{\circ} \mathrm{C}$ to $1300^{\circ} \mathrm{C}$. The thermocouple is suitable for measuring temperature in an inert, reducing, oxidizing and vacuum atmosphere. For accuracy class 1 in the range from $-40^{\circ} \mathrm{C}$ to $375^{\circ} \mathrm{C}$, the temperature deviation is equal to $1.5^{\circ} \mathrm{C}$, and in the range from $375^{\circ} \mathrm{C}$ to $1000^{\circ} \mathrm{C}$ the deviation is $0.4 \%$ [14].

The measuring junction of a thermocouple should be grounded to the metal protective sheath. This results in a short time constant. The protective cover is required to provide protection against mechanical damage to the junction.

Proper selection of material of the outer metal cover provides protection against adverse environmental effects. It also affects the time constant. Inconel 600 (75\% Ni, 16\% $\mathrm{Cr}, 8 \% \mathrm{Fe}$ ) has been deemed the most suitable. It has the following properties [14]:

- good overall corrosion resistance,

- very good oxidation stability,

- the maximum operating temperature in the air of $1150^{\circ} \mathrm{C}$,

- not recommended for an atmosphere above $750^{\circ} \mathrm{C}$ containing sodium.

The diameter of the protective cover influences the thermoelement time constant: the larger the protective cover diameter, the longer time constant. The construction of the dynamic temperature measuring thermocouple used in this research had the smallest protective cover diameter available at the manufacturer that is $0.5 \mathrm{~mm}$, which ensures the time constant of about $8 \mathrm{~ms}$.

The thermocouples were placed on a special trolley that enabled them to move with the engine (Fig. 3). Thanks to this, during each test the temperature measurement was carried out in the same position relative to the engine outlet section.

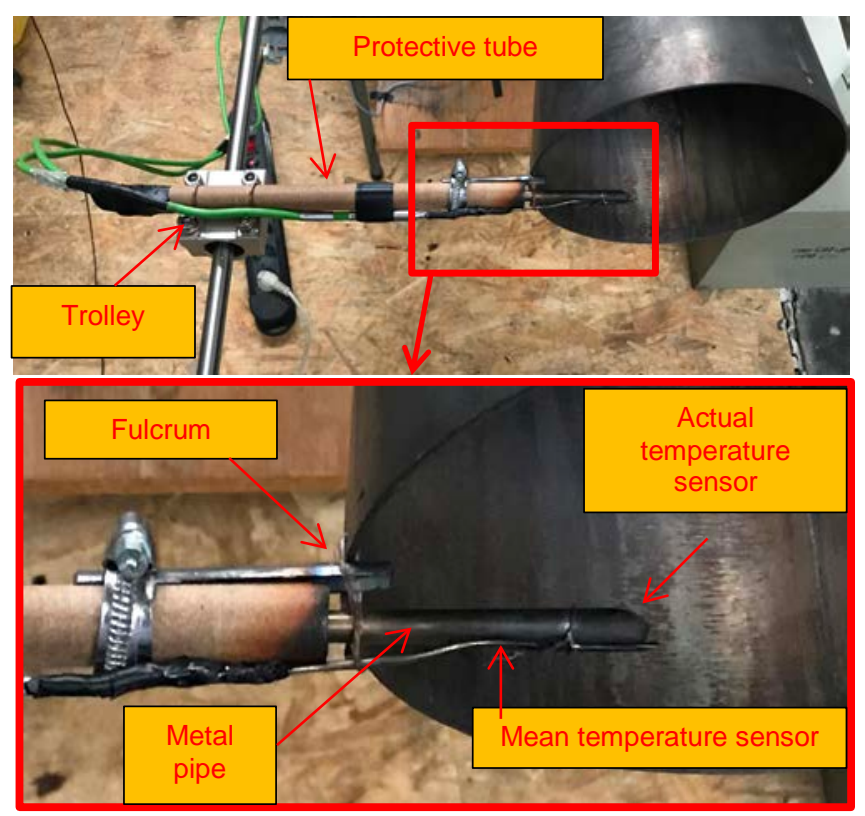

Fig. 3. The view of the system of thermocouples

Due to weak stiffness the actual temperature sensor was routed inside metal pipe of a small diameter to protect it against bending when engine was running. Only the measuring junction was outside the pipe. The mean temperature sensor was routed outside the metal pipe and mechanically connected to it (Fig. 3).

The method of introducing an additional temperature sensor was used to compensate the temperature in the cold junction of the actual thermocouple. It consisted of a PT 100 sensor with an accurate transducer. The solution diagram is shown in Fig. 4. View of the cold end compensation system of the instantaneous thermocouple is shown in Fig. 5. To ensure greater stability of the cold junction temperature, the reference thermocouples together with the resistance sensor were placed in a thick-walled metal tube filled with foamed polystyrene (Fig. 5). The connection of the reference system with the measuring thermocouple was ensured by means of MT-type "socket-plug" terminals (Fig. 5). Due to its small dimensions, the entire compensation system occupied relatively little space on the test stand.

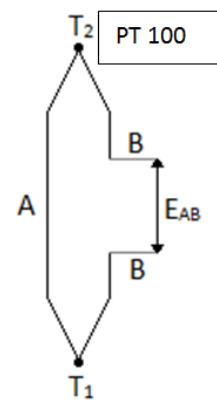

Fig. 4. Schematic diagram of actual temperature measurement system

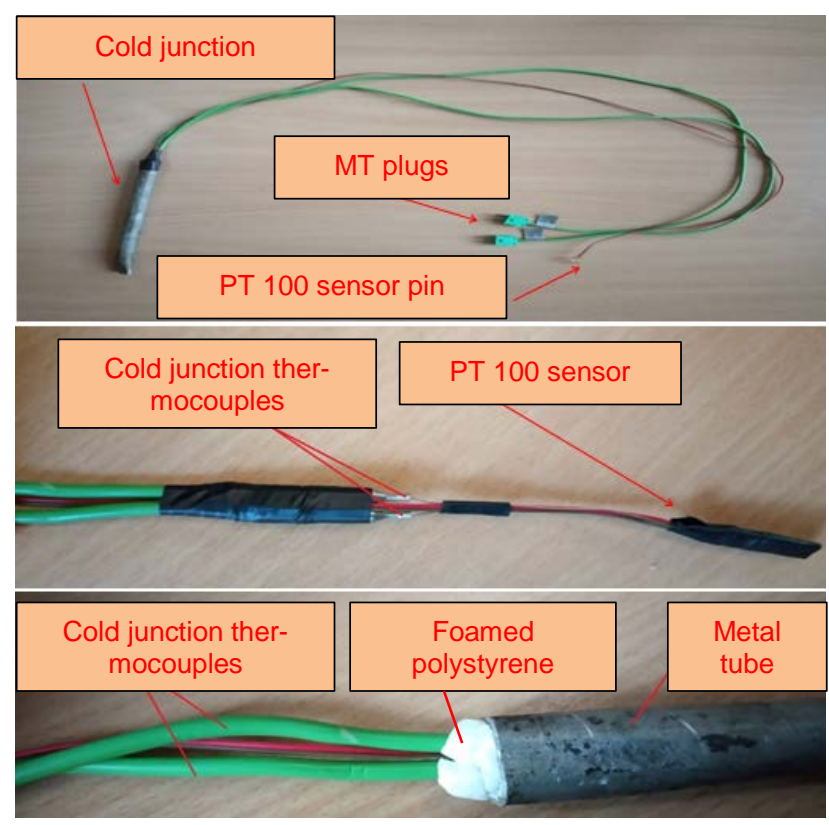

Fig. 5. Construction of actual temperature measurement system

Due to the fact that the thermocouple characteristics are non-linear, the temperature of the cold junction should be converted to Seebeck voltage (E2) in accordance with PN-EN 60584-1. The potential difference between cold and hot junction $\Delta \mathrm{E}$ should be summed with Seebeck voltage E2. The obtained result - the electromotive force E1 should 
be converted into the tested temperature, using the tables contained in PN-EN 60584-1.

$$
\mathrm{E}_{1}=\mathrm{E}_{2}+\Delta \mathrm{E}
$$

Voltage $\Delta \mathrm{E}$ measurement was carried out in a way to meet the law of Intermediate Metals requirements. For this purpose, voltmeter connectors were plugged in MT socket. This place was located away from heat sources, and the signal was transmitted to the measuring card through a shielded cable with a BNC connector at the end. The role of voltmeter was taken by NI 6363 DAQ. The signal from the mean temperature thermocouple was amplified, converted and averaged by a measuring transducer with electronic cold junction compensation.

\subsection{Verification of dynamic temperature measurement system}

During tests the signal from the thermocouple was sampled with frequency of $3500 \mathrm{~Hz}$ and then logged. To verify the proposed method, a spectral analysis of the recorded signal was carried out (Fig. 6).

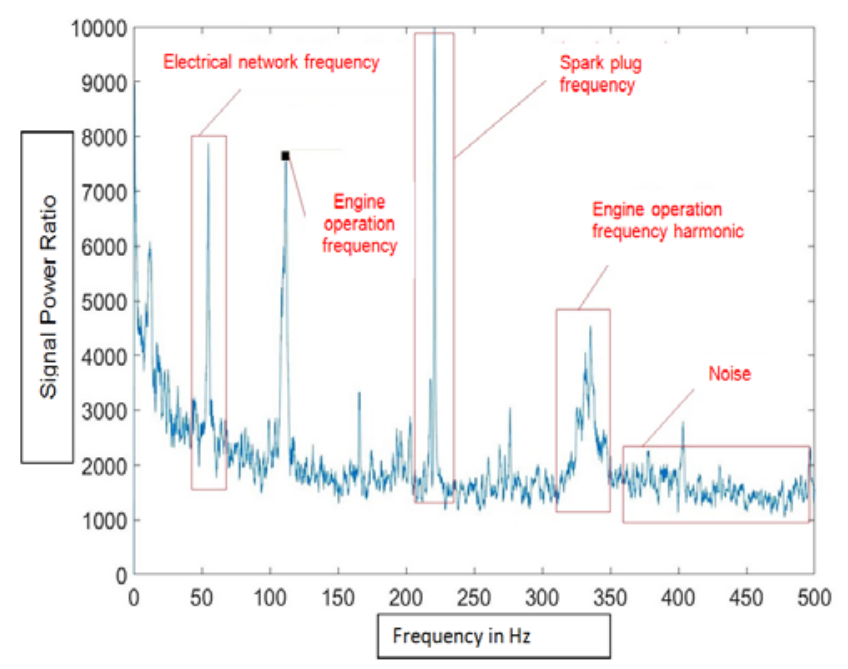

Fig. 6. Spectral analysis of signal from actual temperature thermocouple

Analyzing the spectrum image in detail (Fig. 6) a number of observations about the tested signal can be noted, inter alia it can be deduced that:

- the spectral line for a value of about $55 \mathrm{~Hz}$ is caused by the influence of electrical network interference,

- the spectral line for a value of approx. $230 \mathrm{~Hz}$ is caused by the operation of the spark plug during engine tests,

- for frequencies above $350 \mathrm{~Hz}$, the spectrum is relatively flat,

- the band around $112 \mathrm{~Hz}$ is the frequency of the engine - this coincides with the frequencies determined by other methods.

Engine operating frequency was determined in two independent ways:

- based on spectral analysis of signal of combustion chamber actual pressure,

- based on spectral analysis of sound emitted by the engine.
The results of the evaluation of the engine operating frequency for selected tests are shown in Fig. 7.

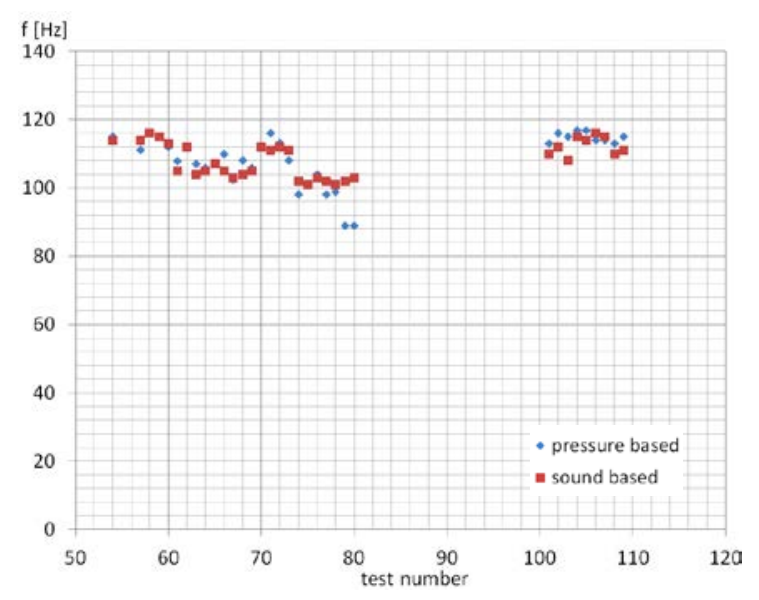

Fig. 7. The value of the engine operating frequency in function of the test number

In Figure 7 it can be seen that the highest concentration of results is in the range of 100-120 Hz. The average engine operating frequency based on pressure waveforms was found to be $108.7 \mathrm{~Hz}$ with a standard deviation of about $7.4 \mathrm{~Hz}$, while for sound analysis it was $108.6 \mathrm{~Hz}$ with a standard deviation of about $5.0 \mathrm{~Hz}$. The differences between the methods are insignificant.

It can be assumed that the main harmonic of the signal generated by the thermocouple was determined by the frequency of engine operation. This was confirmed by spectral analysis of the pressure signal in the combustion chamber and the sound emitted by the engine. For analyzed test the engine operational frequency was equal to $112 \mathrm{~Hz}$. So, it can be deduced that the thermocouple was responding for temperature step function during engine single period. This could lead to conclusion that by using chosen thermocouple it was possible to measure exhaust gases dynamic temperature. A more detailed description of this process will be outlined in chapter 3.3.

\subsection{Determination of the dynamic temperature in the exhaust section}

The temperature measurement in the exhaust section of the pulsejet engine was not balanced. Due to the fact that the sensor was not in the thermal equilibrium with flowing medium it was necessary to take the thermometric lag of the sensor into account. During each test the thermocouple had its own temperature which was different from the medium temperature [12].

According to [12] thermocouple time constant was the time for the sensor to reach 0.632 part of the medium temperature excess over the sensor temperature. It was determined by its manufacturer that a time constant for the type $\mathrm{K}$ thermocouple in question is approx. $8 \mathrm{~ms}$.

The thermocouple response to the violent temperature change provided by the manufacturer is shown in Fig. 8. 


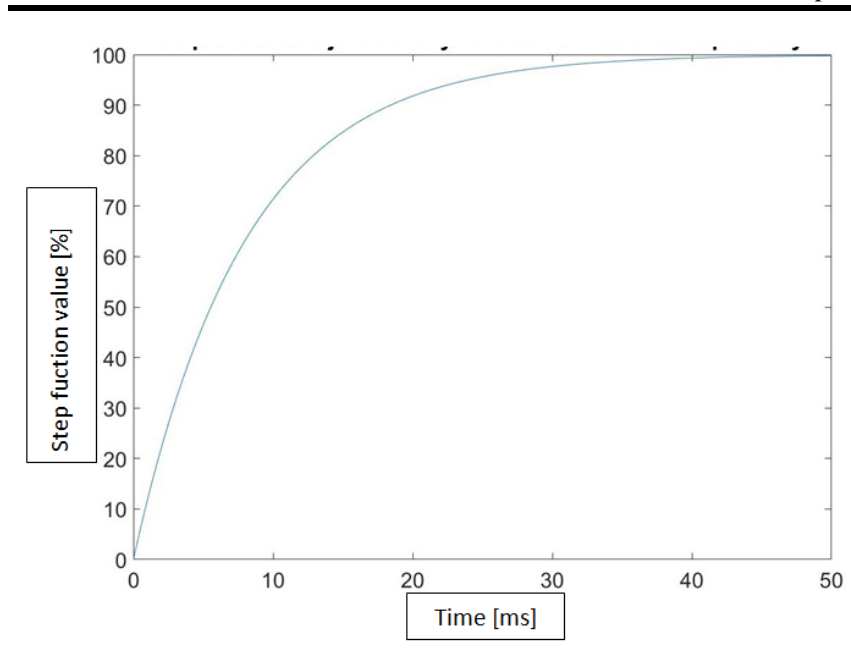

Fig. 8. Sensor response to temperature step function

It can be deduced from the chart shown in Fig. 8 that about 50 ms were needed to equalize the sensor temperature with the medium temperature. In addition, thermocouple time constant was equal to $8 \mathrm{~ms}$, i.e. it was only slightly smaller than a single engine operating period, which is equal to $9 \mathrm{~ms}$. It should be noted that temperature reading obtained from a thermocouple will be different from real temperature of the medium being tested. According to [13] inflow of fresh air to the combustion chamber lasts approximately $40 \%$ of the single cycle time. Due to the fact that the exhaust pipe in this engine was significantly longer than inlet pipes, the inflow of fresh air in the outlet section was even shorter so in consequence the outflow was longer than $60 \%$ of cycle time. Such behavior was a consequence of hot gases mass inertia which was outflowing from the exhaust pipe even after the negative pressure was reached in the combustion chamber [13]. After averaging the pressure and temperature waveforms, it was assumed that for the analyzed test, the gases flow out through the outlet crosssection about $75 \%$ of the entire cycle time. However, the inflow of fresh air took about $25 \%$ of the time of the single cycle. This hypothesis is also consistent with exhaust gases speed record presented in publication [9]. With such outflow to inflow ratios, it was impossible to approximate the temperature course with a sine wave. Since the temperature change in the engine was periodic, it should be remembered that the temperature of the sensor had smaller amplitude and was shifted in phase compared to real temperature course. From [12] it follows that for sine input function sensor amplitude is equal to:

$$
\mathrm{T}_{\mathrm{a}}=\frac{\mathrm{T}_{\mathrm{ga}}}{\sqrt{1+\omega^{2} \tau^{2}}}
$$

Phase shift is equal to:

$$
\varphi=\operatorname{arctg}(\omega \tau)
$$

where $T_{a}$ - is the pulsation temperature amplitude, $T_{g a}-$ mean temperature of media $\omega$ - frequency of pulsation, $\tau-$ sensor time constant, $\varphi$ - phase shift between real temperature course and sensor temperature.

An exemplary temperature measurement in the outlet section was carried out for the following conditions:

- an ambient temperature of $20^{\circ} \mathrm{C}$,
- fuel mass flow rate $4.5 \mathrm{~g} / \mathrm{s}$.

After using a bandpass filter with an $80-160 \mathrm{~Hz}$ transmission bandwidth, the dynamic temperature course from the sensor was obtained (Fig. 9). The temperature course of the sensor presented in Figure 9 is not equal to the actual engine outlet temperature. Since the analyzed waveform is not a sine wave, the relationship between the medium temperature and the sensor temperature proposed in publication [12] does not give the expected result. This will be detailed further in the text.

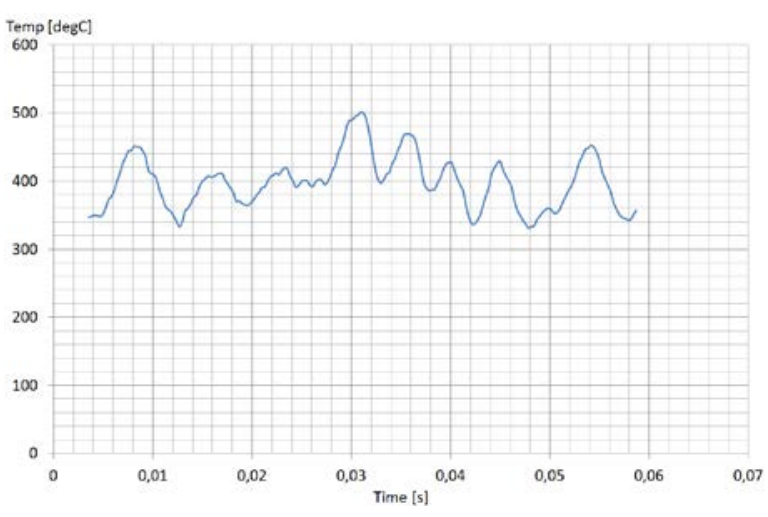

Fig. 9. The actual temperature sensor signal course

Since the estimation of the temperature course in the outlet section of the pulse jet engine by analytical method did not give satisfactory results, simulations of thermocouple behavior in given operating conditions were carried out. For the purpose of the simulation it was assumed that:

- engine operational frequency is equal to $112 \mathrm{~Hz}$,

- the outflow from outlet station takes $75 \%$ of total time of single cycle time,

- the inflow through outlet station takes $25 \%$ of total time of single cycle time,

- the temperature of incoming air is equal to an ambient temperature of $20^{\circ} \mathrm{C}$,

- the exhaust gases temperature - iterated variable.

During the subsequent iterations for given operational conditions the exhaust gases temperature was changed to reach the closest match between simulated and registered signal. To make simulation as close to reality as possible the behavior of mean temperature sensor was iterated in parallel. The resultant simulated course for dynamic temperature sensor is presented in Fig. 10.

In Figure 10 first simulated point corresponds to temperature which sensor reached after the outflow in first engine period. The second point corresponds to the end of inflow. From the simulated course it can be deduced that after about $35 \mathrm{~ms}$ the sensor was thermally stabilized.

Due to unstable work of the engine directly after the start up, only sections when the thermocouple was thermally stabilized were chosen for comparison. The comparison of simulated and real sensor behavior is presented in Fig. 11.

The presented comparison shows that the amplitudes of the real wave are slightly smaller than the simulated ones. This may be due to the fact that the air that was drawn in by the engine had a slightly higher temperature during its operation as a result of heating the environment with hot ex- 
haust fumes. Secondly, the real waveform has a very irregular shape and is strongly distorted.

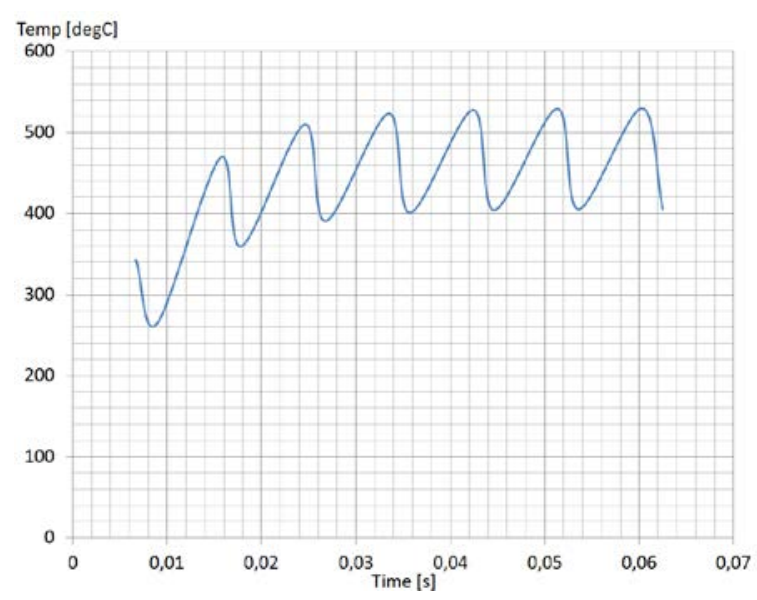

Fig. 10. Simulated signal course from instantaneous temperature sensor

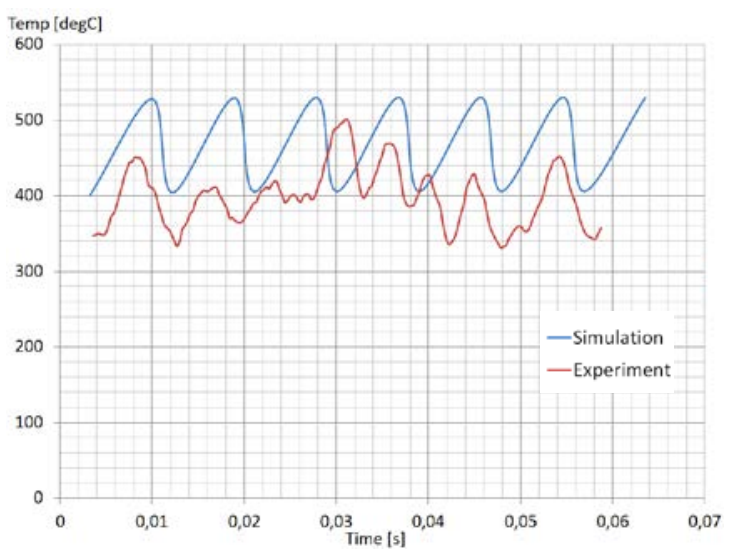

Fig. 11. Comparison of simulated and real instantaneous temperature sensor behavior

From Fig. 11 it could be also deduced that times of rise and decay are similar, what might be treated as further confirmation of assumed outflow to inflow relation, as well as confirms that the time constant taken to the simulation is appropriate.

Despite of number measures taken to improve the quality of signal from the sensor, the signal distortions were still present. This was mainly caused by the fact that the thermocouple measures temperature at one point and local flow fluctuations produces the random noise. What is more, in the outlet station of the engine a flame could be observed which resulted in further distortions of sensor readings due to ionization of air in the vicinity of the sensor. It is not possible to completely remove distortions from the analyzed waveform. Especially, the random noise mentioned above. However, the presented accuracy should be sufficient to estimate the maximum exhaust gas temperature. In the discussed case, it was around $625^{\circ} \mathrm{C}$ which gives an amplitude of $\mathrm{A}=605^{\circ} \mathrm{C}$. When assuming a sinusoidal course of temperature changes, according to publication [12], the sensor amplitude calculated on the base of equation (2) should be around $450^{\circ} \mathrm{C}$. This value is far from reality and confirms the statement that formula (2) is inadequate for the case considered. The course of the iterated real, simulated and thermocouple measured signal curves are shown in Fig. 12.

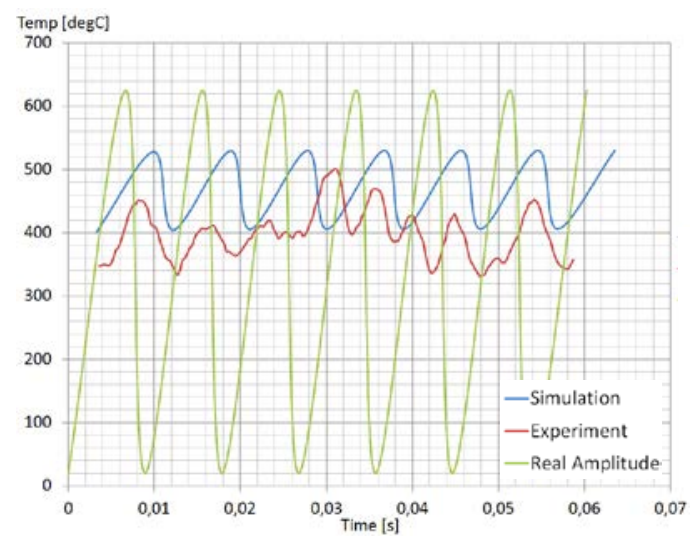

Fig. 12. Comparison of simulated and real sensor behavior with estimated real temperature course

In Figure 12 between real temperature course and thermocouple signals a phase shift calculated accordingly to equation 3 was applied. According to calculations, the temperature of the sensor reaches its maximum after $3.3 \mathrm{~ms}$ from reaching the maximum temperature in the outlet station, which is about $1 / 3$ of the engine's single cycle time. As can be easily noticed in Fig. 12, the temperature of the sensor increases when the inflow of fresh air through the outlet station begins and decreases when the outflow of hot gases continues. This arrangement of the waveforms may suggest that the formula used to calculate the phase shift is inadequate or there are some errors in the initial assumptions of the simulation.

Firstly, the real temperature changes can be more violent than assumed. This could be due to the fact that hot gases leave the exhaust pipe in isothermal portions that flow through the outlet section for some time. In simulation it was assumed that it is one point with infinitely short duration. It can be similar in the inflow cycle. A portion of air with a constant temperature higher than assumed in the simulation can be sucked in. This may be due to heating the ambient air in the vicinity of outlet station by hot fumes what was described earlier in the text.

In the next drawing (Fig. 13) the simulation results with real waveform of mean temperature sensor are compared.

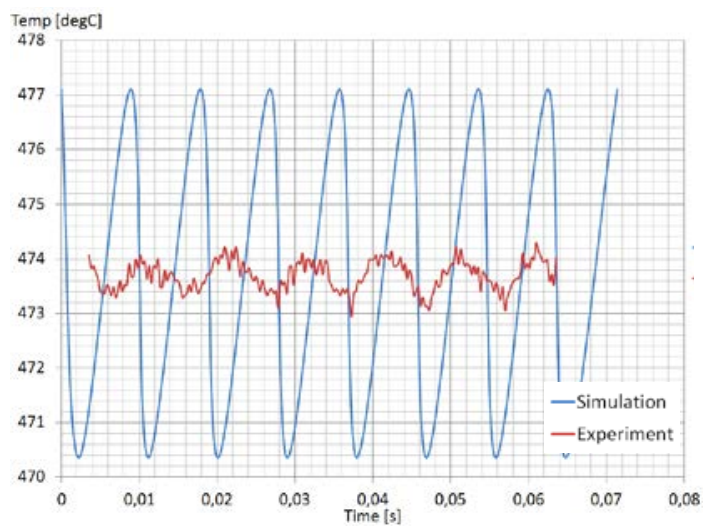

Fig. 13. Comparison of simulated and real average temperature sensor behavior 
The simulated thermocouple waveform shown in Fig. 13 has a significantly greater amplitude than the real course. This can further confirm the fact that the temperature of the air sucked through the outlet is higher than the ambient temperature. However, both waveforms are consistent in terms of pattern and frequency. Both waveforms also oscillate around the same $474^{\circ} \mathrm{C}$ value. The simulated and recorded waveform for thermocouple with a $8 \mathrm{~ms}$ time constant also oscillates around this value. This may indicate that, despite the simplifications adopted, it was possible to correctly estimate the true temperature changes in the outlet section of the pulsejet engine.

\section{Summary}

Temperature measurement in the outlet station of a pulsejet engine is a complicated task. This undertaking is hampered by such properties of the tested object as the explosive nature of the combustion process causing large fluctuations in flow velocity, vibrations of the structure and acoustic effects, as well as the lack of generally available sensors that would allow direct measurement of temperature oscillations in the outlet section of the tested engine.

A significant difference in the duration of the outflow phase and the inflow of air through the outlet station results in the inability to use known and proven relationships to determine the real temperature of the medium being tested. This is due to the fact that the course of temperature changes cannot be approximated by a sine wave. In addition, it is not possible to describe the actual temperature as a continuous function of time. It also translates into the inability to analytically determine the RMS or average value of the analyzed waveform.

The aim of the presented works was to determine EGT in the outlet section of a pulsejet. Its maximum value and frequency of change is particularly useful for further analysis. The method to achieve this goal should be simple and quick so that EGT can be estimated after each specific test, without causing significant breaks in work. Therefore, it was decided to develop an iterative EGT method. The method is rough, and the results should be treated as preliminary.

Iterating of the maximum temperature values in the outlet section proved to be the effective approach to the problem. Thanks to this, it was possible to determine the approximate value of exhaust gases temperature. Due to applied simplifications however, this result is also subject to uncertainty. Despite this, at the current level of work, the obtained accuracy seems to be sufficient.

\section{Bibliography}

[1] ARJAVALINGAM, G., HAYNES, R.D., HYER, G.N. et al. High-temperature thin-film Pt-Ir thermocouple with fast time response. Review of Scientific Instruments. 1987, 58(5), 875877. https://doi.org/10.1063/1.1139649

[2] BORDATCHEV, E., CHANDRA, S., HEICHAL, Y. A fastresponse thin film thermocouple to measure rapid surface temperature changes. Experimental Thermal and Fluid Science. 2005, 30(2), 153-159.

https://doi.org/10.1016/j.expthermflusci.2005.05.004

[3] CHEN, L., FRALICK, G., GREER, L. et al. Design and operation of a fast, thin-film thermocouple probe on a turbine engine. $50^{\text {th }}$ AIAA/ASME/SAE/ASEE Joint Propulsion Conference, NASA Center for AeroSpace Information (CASI). Conference Proceedings. 2014.

https://doi.org/10.2514/6.2014-3923

[4] DE JONG, W., KUDRA, T., MENG, X. A state-of-the-art review of pulse combustion: Principles, modeling, applications and R\&D issues. Renewable and Sustainable Energy Reviews.. 2016, 55, 73-114.

https://doi.org/10.1016/j.rser.2015.10.110

[5] FLECK, B., GAYNOR, J., HUNG, P. et al. Fast response exhaust gas temperature measurement in IC engines. $S A E$ Transactions. Journal of Passenger Cars: Electronic and Electrical Systems. 2006, 115(7), 598-609.

[6] FLECK, R., KEE, R.J., MCENTEE, P.T. et al. Measurement of exhaust gas temperatures in a high performance two-stroke

Adrian Trzeciak, MEng.. - Faculty of Power and Aeronautical Engineering, Warsaw University of Technology.

e-mail: adrian.trzeciak.dokt@pw.edu.pl

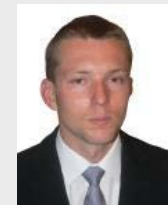

engine. SAE Transactions. Journal of Engines. 1998, 107(3). 2413-2423.

[7] HROMASOVA, M., LINDA, M. Analysis of rapid temperature changes. Agronomy Research. 2016, 14(3), 768-778.

[8] LING, Y., MIN, L., WEN, C. Experimental analysis of combustion performance in pulse jet engine. Energy Procedia. 2016, 100, 248-252.

[9] POBEZHIMOW, V.N. Flight efficiency of a pulsejet. Russian Aeronautics. 2010, 53(1), 77-80.

https://doi.org/10.3103/S1068799810010137

[10] Pulsating Combustion. International Symposium. Monterey 1991. Print. Combustion Science and Technology. 1993, 1(6).

[11] THOMA, K. Dynamic temperature measurements on a thermally activated self-healing ionomer. Journal of Intelligent Material Systems and Structures. 2014, 25(1), 25-30. https://doi.org/10.1177/1045389X12444487

[12] WIŚNIEWSKI, S. Pomiary temperatury w badaniach silników i urządzeń cieplnych. Wydawnictwo NaukowoTech-niczne Warszawa 1983.

[13] WÓJCICKI, S. Silniki pulsacyjne, strumieniowe, rakietowe. Wydawnictwo Ministerstwa Obrony Narodowej. Warszawa 1962.

[14] https://www.czaki.pl/content-dir/uploads/czakiczujniki_termoparowe.pdf; accessed 02.2020.

Prof. Marian Gieras, DSc., DEng. - Faculty o Power and Aeronautical Engineering, Warsaw University of Technology.

e-mail: marian.gieras@pw.edu.pl

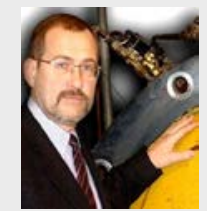

